\title{
Chemical coding of zinc-enriched neurons in the intramural ganglia of the porcine jejunum
}

\author{
Joanna Wojtkiewicz • Maciej Równiak • \\ Robert Crayton • Mariusz Majewski • \\ Slawomir Gonkowski
}

Received: 18 April 2012 / Accepted: 2 August 2012 / Published online: 24 August 2012

(C) The Author(s) 2012. This article is published with open access at Springerlink.com

\begin{abstract}
Zinc ions in the synaptic vesicles of zinc-enriched neurons (ZEN) seem to have an important role in normal physiological and pathophysiological processes in target organ innervation. The factor directly responsible for the transport of zinc ions into synaptic vesicles is zinc transporter 3 (ZnT3), a member of the divalent cation zinc transporters and an excellent marker of ZEN neurons. As data concerning the existence of ZEN neurons in the small intestine is lacking, this study was designed to disclose the presence and neurochemical coding of such neurons in the porcine jejunum. Cryostat sections ( $10 \mathrm{~m} \mu$ thick) of porcine
\end{abstract}

This work was supported by grant no. NN401178639 from the State Committee for Science Research of Poland.

\footnotetext{
J. Wojtkiewicz $(\bowtie)$

Faculty Medical Sciences, Department of Neurology

and Neurosurgery, Stem Cell Research Laboratory,

University of Warmia and Mazury,

ul. Warszawska 30,

10-082 Olsztyn, Poland

e-mail: Joanna.Wojtkiewicz@uwm.edu.pl

M. Równiak

Department of Comparative Anatomy, Faculty of Biology,

University of Warmia and Mazury,

Olsztyn, Poland

R. Crayton

Department and Clinic of Urology, Faculty of Medical Sciences,

Medical University of Warsaw,

Warsaw, Poland

M. Majewski

Department of Human Physiology, Faculty of Medical Sciences,

University of Warmia and Mazury,

Olsztyn, Poland

S. Gonkowski

Department of Clinical Physiology, Faculty of Veterinary

Medicine, University of Warmia and Mazury,

Olsztyn, Poland
}

jejunum were processed for routine double- and tripleimmunofluorescence labeling for ZnT3 in various combinations with immunolabeling for other neurochemicals including pan-neuronal marker (PGP9.5), substance P (SP), somatostatin (SOM), vasoactive intestinal peptide (VIP), nitric oxide synthase (NOS), leu-enkephalin (LENK), vesicular acetylcholine transporter (VAChT), neuropeptide $\mathrm{Y}$ (NPY), galanin (GAL), and calcitonin-gene related peptide (CGRP). Immunohistochemistry revealed that approximately $39 \%, 49 \%$, and $45 \%$ of all PGP9.5- positive neurons in the jejunal myenteric (MP), outer submucous (OSP), and inner submucous (ISP) plexuses, respectively, were simultaneously $\mathrm{ZnT}^{+}$. The majority of $\mathrm{ZnT}^{+}$neurons in all plexuses were also VAChT-positive. Both VAChT-positive and VAChT-negative $\mathrm{ZnT}^{+}$neurons co-expressed a variety of active substances with diverse patterns of co-localization depending on the plexus studied. In the MP, the largest populations among both VAChT-positive and VAChTnegative $\mathrm{ZnT}^{+}$neurons were NOS-positive cells. In the OSP and ISP, substantial subpopulations of $\mathrm{ZnT}^{+}$neurons were VAChT-positive cells co-expressing SOM and GAL, respectively. The broad-spectrum of active substances that co-localize with the $\mathrm{ZnT}^{+}$neurons in the porcine jejunum suggests that $\mathrm{ZnT} 3$ takes part in the regulation of various processes in the gut, both in normal physiological and during pathophysiological processes.

Keywords Zinc-enriched neurons · Jejunum · Small intestine $\cdot$ Immunolabeling technique $\cdot$ Enteric nervous system $\cdot$ Domestic pig

\section{Introduction}

Recent studies on the chemical coding of the enteric nervous system (ENS) have identified a subpopulation of neurons 
that express the zinc transporter 3 (ZnT3; Gonkowski et al. 2009; Gonkowski 2011). ZnT3 is a member of the SLC 30 zinc transporter family, which is responsible for transport between extracellular, cytoplasmic, and intracellular organelle compartments (Palmiter and Huang 2004). Importantly, the ZnT3-mediated transport of zinc into synaptic vesicles serves to modulate neuron activity (Palmiter et al. 1996) and thus plays an important role in the normal physiological and pathophysiological changes of the nervous system (Frederickson et al. 2000, 2005; Smart et al. 2004). Zincenriched nerve (ZEN) terminals that utilize ZnT3 have been found in the hippocampus, amygdala, neocortex, spinal cord, and superior cervical ganglion neurons (Jo et al. 2000; Wang et al. 2003; Wenzel et al. 1997). Since previous studies on the central nervous system (CNS) have suggested that $\mathrm{ZnT3}$ is present in neurons that use $\mathrm{Zn}$ as neuromodulator (Cousins et al. 2006; Danscher et al. 2003; Kim et al. 2000; Wang et al. 2003; Wenzel et al. 1997), ZnT3 can therefore be used as a marker for tracing ZEN structures. Although the role of $\mathrm{Zn}$ as a neuromodulator/neurotransmitter in the nervous system remains obscure, convincing evidence suggests that this cation is a potent modulator of various receptors and several transporters in the brain, and that it thereby influences both excitatory and inhibitory neurotransmission (Betz and Laube 2006; Frederickson et al. 2005; Smart et al. 2004). The presence of $\mathrm{Zn}$ in the ENS might suggest a similar modulatory role.

In humans and pigs, the ENS is organized into three plexuses: myenteric plexus (MP), outer submucous plexus (OSP), and inner submucous plexus (ISP). All these plexuses are populated with above 20 classes of neurons with different functions and targets and a large variety of neurotransmitters (Brown and Timmermans 2004; Furness 2000). Until now, only two studies have reported the presence of $\mathrm{ZnT3}$ in the ENS. However, both of these studies have centered on the large intestine (Gonkowski et al. 2009; Gonkowski 2011). At present, no data on other parts of the gastrointestinal tract are available. Therefore, the aim of the present study has been to investigate the distribution, number, and chemical coding pattern of $\mathrm{ZnT}^{+}$neurons in the intramural ganglia of the porcine jejunum.

\section{Materials and methods}

Study subjects

Six juvenile female pigs ( $8-10$ weeks, $12-15 \mathrm{~kg}$ body weight) of the Large White Polish breed were used. All animals were housed and treated in accordance with the Principles of Laboratory Animal Care (NIH publication no. 86-23, revised 1985). All experimental procedures were approved by the Local Ethics Commission of the University of Warmia and Mazury in Olsztyn (no. 27/2009).

Anesthesia and surgery

All animals were pretreated with atropine sulfate (Polfa, Poland; $0.04 \mathrm{mg} / \mathrm{kg}$ body weight, s.c.) and azaperone (Stressnil, Janssen Pharmaceutica, Belgium; $2.0 \mathrm{mg} / \mathrm{kg}$ body weight, i.m.) $30 \mathrm{~min}$ prior to administration of the main anesthetic. Surgery was performed under fractionated thiobarbital (Thiopenthal, Sandoz, Austria; $20 \mathrm{mg} / \mathrm{kg}$ b.w., i.v.) anesthesia. All animals were killed by overdoses of thiobarbital and perfused transcardially with $4 \%$ buffered paraformaldehyde ( $\mathrm{pH} 7.4)$. Following perfusion, the jejunum from all the animals was dissected out, cut into tissue blocks, postfixed by immersion in the same fixative for $4 \mathrm{~h}$, washed twice in $0.1 \mathrm{M}$ phosphate buffer $\left(p H 7.4,4^{\circ} \mathrm{C}\right)$ for 3 days, and then stored in $18 \%$ sucrose at $4^{\circ} \mathrm{C}$ until sectioned. Other organs from these animals were also collected for further research in our or other laboratories.

\section{Immunofluorescence experiments}

Jejunum samples were cut into sections $(10 \mu \mathrm{m}$ thick) by using a cryostat (Hyrax C25; Carl Zeiss, Germany) and processed for double- and triple-immunofluorescence. All samples were washed three times in phosphate-buffered saline (PBS) and then incubated in humid chambers with blocking buffer $(0.1 \mathrm{M}$ PBS, $10 \%$ normal horse serum, $0.01 \%$ bovine serum albumin, $1 \%$ Tween, $0.05 \%$ thimerosal, $0.01 \% \mathrm{NaN}_{3}$ ) for $1 \mathrm{~h}$. The sections were then rinsed in PBS and incubated overnight at room temperature with a mixture of primary antibodies, namely a combination of the appropriate primary antisera to zinc transporter 3 (ZnT3), pan-neuronal marker (PGP9.5), substance P (SP), somatostatin (SOM), vasoactive intestinal peptide (VIP), nitric oxide synthase (NOS), leu-enkephalin (LENK), vesicular acetylcholine transporter (VAChT), neuropeptide Y (NPY), galanin (GAL), and calcitonin-gene related peptide (CGRP; Table 1). After incubation with the primary antibodies, the sections were rinsed in PBS and incubated for $1 \mathrm{~h}$ with biotinylated secondary antibodies (during double-labeling immunofluorescence) or with biotinylated 7-amino-4-methylcoumarin-3-acetic acid (AMCA; during triple-labeling immunofluorescence; Table 1). After $1 \mathrm{~h}$, the sections were finally incubated with a mixture of fluorescein isothiocyanate (FITC) and CY3-conjugated streptavidin (Table 1). Finally, all samples were rinsed in PBS and then mounted with carbonate-buffered glycerol (pH 8.6) and coverslipped.

\section{Controls}

Standard controls, i.e., preabsorption for the neuropeptide antisera (20 mg appropriate antigen per $1 \mathrm{ml}$ corresponding 
Table 1 Specification of immune reagents vs. zinc transporter 3 (PGP9.5 pan-neuronal marker, ZnT3 zinc transporter 3, NOS nitric oxide synthase, VIP vasoactive intestinal peptide, $S P$ substance $\mathrm{P}$, $S O M$ somatostatin, $L E N K$ leu-enkephalin, VAChT vesicular acetylcholine transporter, $N P Y$ neuropeptide $\mathrm{Y}, G A L$ galanin, $C G R P$ calcitonin-gene related peptide, FITC fluorescein isothiocyanate, $A M C A$ 7-amino-4-methylcoumarin-3-acetic acid, $H$ heavy chain, $L$ light chain)

\begin{tabular}{|c|c|c|c|c|}
\hline Antisera & Code & Host species/specificity & Dilution & Supplier \\
\hline \multicolumn{5}{|l|}{ Primary antibody } \\
\hline PGP9.5 & $7863-2004$ & Mouse & $1: 2000$ & Biogenesis, UK; \\
\hline $\mathrm{ZnT3}$ & - & Rabbit & $1: 600$ & Gift from Prof. Palmiter, USA \\
\hline NOS & $\mathrm{N} 2280$ & Mouse & 1: 2000 & Sigma, US; www.sigma-aldrich.com \\
\hline VIP & $9535-0504$ & Mouse & 1: 2000 & \multirow[t]{4}{*}{ Biogenesis } \\
\hline SP & $8450-0505$ & Rat & $1: 300$ & \\
\hline SOM & $8330-0009$ & Rat & 1: 100 & \\
\hline LENK & $4140-0355$ & Mouse & 1: 1000 & \\
\hline VAChT & $\mathrm{H}-\mathrm{V} 007$ & Goat & 1: 2000 & $\begin{array}{l}\text { Phoenix, Pharmaceuticals, US; } \\
\text { www.phoenixpeptide.com }\end{array}$ \\
\hline NPY & NZ1115 & Rat & $1: 300$ & Biomol Research Laboratories, US \\
\hline $\begin{array}{l}\text { GAL } \\
\text { CGRP }\end{array}$ & $\begin{array}{l}\mathrm{T}-5036 \\
\mathrm{~T}-5027\end{array}$ & $\begin{array}{l}\text { Guinea pig } \\
\text { Guinea pig }\end{array}$ & $\begin{array}{l}1: 1000 \\
1: 1000\end{array}$ & $\begin{array}{l}\text { Peninsula Labs, US; see Bachem; } \\
\text { www.bachem.com }\end{array}$ \\
\hline \multicolumn{5}{|l|}{ Secondary antibodies } \\
\hline \multirow[t]{3}{*}{ FITC-conjugated $\operatorname{IgG}(\mathrm{H}+\mathrm{L})$} & $\begin{array}{l}715-095-151 \\
712-095-153\end{array}$ & $\begin{array}{l}\text { Donkey-anti-mouse } \\
\text { Donkey-anti-rat }\end{array}$ & $\begin{array}{l}1: 800 \\
1: 800\end{array}$ & \multirow[t]{3}{*}{ Jackson } \\
\hline & 706-095-148 & Donkey-anti-guinea pig & $1: 1000$ & \\
\hline & $705-096-147$ & Donkey-anti-goat & $1: 1000$ & \\
\hline Biotinylated IgGs & Е 0432 & Goat anti-rabbit & $1: 1000$ & DAKO, E 0432 \\
\hline $\begin{array}{l}\text { Biotin conjugated } \mathrm{F}(\mathrm{ab})^{\prime} \text { fragment of } \\
\text { affinity-purified } \operatorname{IgG}(\mathrm{H}+\mathrm{L})\end{array}$ & $711-1622$ & Anti-rabbit & $1: 1000$ & BioTrend, 711-1622 \\
\hline \multirow[t]{2}{*}{ AMCA-conjugated $\operatorname{IgG}(\mathrm{H}+\mathrm{L})$} & $\begin{array}{l}715-155-151 \\
715-155-153\end{array}$ & $\begin{array}{l}\text { Donkey-anti-mouse } \\
\text { Donkey-anti-rat }\end{array}$ & $\begin{array}{l}1: 50 \\
1: 50\end{array}$ & \multirow[t]{2}{*}{ Jackson } \\
\hline & $705-156-147$ & Donkey-anti-goat & $1: 50$ & \\
\hline CY3-conjugated streptavidin & 016-160-084 & - & 1:9000 & \\
\hline
\end{tabular}

antibody at working dilution) and the omission and replacement of all primary antisera by non-immune sera or PBS, were applied to test both antibody and method specificity.

Counts and statistics

The sections were observed by using an Olympus BX51 fluorescence microscope equipped with epi-fluorescence and an appropriate filter set for FITC, CY3, and AMCA. Microphotographs were acquired with Cellsens Olympus image analysis software (ver. 3.2; Soft Imaging System, Münster, Germany). To determine the percentages of $\mathrm{ZnT}^{+}$neurons in each plexus studied (MP, OSP, and ISP), pan-neuronal marker PGP-9.5 was adopted. PGP-9.5 marks all neurons in the tissue, and so $\mathrm{PGP}^{+} / \mathrm{ZnT}^{+}$cells illustrate the percentages of MP, OSP, and ISP neurons co-expressing ZnT3. At least 1000 PGP9.5-labeled cell bodies located in 60-70 ganglia of a particular plexus (MP, OSP, and ISP) per animal were examined for ZnT3 immunoreactivity. Only neurons with clearly visible nuclei were counted. To prevent double-counting of $\mathrm{ZnT}^{+}$neurons, the sections were located at least $200 \mu \mathrm{m}$ apart from each other. Moreover, to determine the percentages of colocalization of ZnT3 with other substances studied, at least 700 ZnT3-positive cell bodies in particular types of enteric plexuses were examined for immunoreactivity to the particular substances investigated. In these double- and triple-labeling studies, ZnT3-positive neurons were considered as representing $100 \%$ for all combinations, and so all the values shown in the text and Table 2 are percentages of $\mathrm{ZnT}^{+}$neurons. Finally, data were pooled from all 6 animals and expressed as means \pm SD and then analyzed by using GraphPad Prism 5 software (GraphPad Software, La Jolla, Calif., USA).

\section{Results}

Distribution and number of $\mathrm{ZnT}^{+}$neurons in porcine jejunum

$\mathrm{ZnT}^{+}$cell bodies were observed in all enteric plexuses within porcine jejunum, i.e., in the MP (located between the longitudinal and circular muscle layers), the OSP (found in the 
Table 2 Neurochemical characterization of zinc-transporter-3-immunoreactive $\left(\mathrm{ZnT}^{+}\right)$neurons in the enteric ganglia of the porcine jejunum (MP myenteric plexus, OSP outer submucosal plexus, ISP inner submucosal plexus, $s$ single neurons). Note that PGP9.5 is a panneuronal marker that marks all neurons in the tissue, and so $\mathrm{PGP}^{+}$/ $\mathrm{ZnT}^{+}$cells illustrate the percentages of MP, OSP, and ISP neurons coexpressing ZnT3. In the triple-labeling studies, ZnT3-positive neurons were considered as representing $100 \%$ for all combinations with other neurotransmitters, and so all the values presented are percentages (means $\pm \mathrm{SD}$ ) of $\mathrm{ZnT}^{+}$neurons

\begin{tabular}{llll}
\hline Labeling & MP & OSP & ISP \\
\hline $\mathrm{PGP}^{+} / \mathrm{ZnT3}^{+}$ & $38.6 \pm 4.0$ & $48.7 \pm 10.8$ & $44.8 \pm 4.1$ \\
$\mathrm{ZnT3}^{+} / \mathrm{VAChT}^{-}$ & $39.2 \pm 5.6$ & $5.8 \pm 1.2$ & $2.8 \pm 1.0$ \\
$\mathrm{ZnT3}^{+} / \mathrm{VAChT}^{-} / \mathrm{NOS}^{+}$ & $37.7 \pm 5.4$ & $4.1 \pm 1.8$ & 0 \\
$\mathrm{ZnT}^{+} / \mathrm{VAChT}^{-} / \mathrm{VIP}^{+}$ & $5.8 \pm 3.4$ & $1.3 \pm 0.9$ & $2.1 \pm 0.9$ \\
$\mathrm{ZnT3}^{+} / \mathrm{VAChT}^{-} / \mathrm{SOM}^{+}$ & $5.4 \pm 1.2$ & $2.2 \pm 0.8$ & $1.1 \pm 0.8$ \\
$\mathrm{ZnT3}^{+} / \mathrm{VAChT}^{-} / \mathrm{SP}^{+}$ & $2.5 \pm 1.3$ & $0.9 \pm 0.5$ & $1.5 \pm 0.7$ \\
$\mathrm{ZnT3}^{+} / \mathrm{VAChT}^{-} / \mathrm{LENK}^{+}$ & $1.2 \pm 0.6$ & 0 & 0 \\
$\mathrm{ZnT3}^{+} / \mathrm{LENK}^{+} / \mathrm{SP}^{+}$ & $\mathrm{s}$ & 0 & 0 \\
$\mathrm{ZnT3}^{+} / \mathrm{VAChT}^{-} / \mathrm{GAL}^{+}$ & 0 & $\mathrm{~s}$ & $2.0 \pm 0.8$ \\
$\mathrm{ZnT3}^{+} / \mathrm{VAChT}^{-} / \mathrm{NPY}^{+}$ & $\mathrm{s}$ & 0 & 0 \\
$\mathrm{ZnT3}^{+} / \mathrm{VAChT}^{-} / \mathrm{CGRP}^{+}$ & 0 & 0 & 0 \\
$\mathrm{ZnT3}^{+} / \mathrm{VAChT}^{+}$ & $60.8 \pm 5.6$ & $94.2 \pm 1.2$ & $97.2 \pm 4.3$ \\
$\mathrm{ZnT3}^{+} / \mathrm{VAChT}^{+} / \mathrm{SOM}^{+}$ & $2.2 \pm 1.2$ & $41.1 \pm 1.8$ & $27.2 \pm 5.4$ \\
$\mathrm{ZnT3}^{+} / \mathrm{VAChT}^{+} / \mathrm{VIP}^{+}$ & $1.1 \pm 0.3$ & $21.9 \pm 1.3$ & $30.9 \pm 2.3$ \\
$\mathrm{ZnT3}^{+} / \mathrm{VAChT}^{+} / \mathrm{SP}^{+}$ & $2.5 \pm 1.3$ & $16.1 \pm 2.8$ & $28.4 \pm 1.9$ \\
$\mathrm{ZnT3}^{+} / \mathrm{VAChT}^{+} / \mathrm{GAL}^{+}$ & 0 & 0 & $47.1 \pm 1.9$ \\
$\mathrm{ZnT3}^{+} / \mathrm{VAChT}^{+} / \mathrm{NOS}^{+}$ & $10.4 \pm 3.1$ & $3.7 \pm 1.1$ & 0 \\
\hline
\end{tabular}

submucosa), and the ISP (located on the abluminal side of the muscularis mucosae). The proportions of $\mathrm{ZnT}^{+}$neurons varied depending on the plexus studied, being the largest within the OSP and the smallest in the MP; however, these differences between percentages were not statistically significant (Table 2, Fig. 1a-c). No $\mathrm{ZnT}^{+}$nerve fibers were observed in the porcine jejunum.

Co-localization pattern of $\mathrm{ZnT}^{+}$neurons in porcine jejunum

In all plexuses, the population of $\mathrm{ZnT}^{+}$neurons could be subdivided into cholinergic $\left(\mathrm{Znt}^{+} / \mathrm{VAChT}^{+}\right)$and noncholinergic $\left(\mathrm{Znt}^{+} / \mathrm{VAChT}^{-}\right)$neurons. Both cholinergic and non-cholinergic $\mathrm{Znt}^{+}$cells co-expressed a broadspectrum of the other substances tested in the present study, but the co-localization patterns were different in each of the plexus studied (Table 2). The only substance that was never co-expressed in $\mathrm{ZnT}^{+}$neurons was CGRP (Table 2).

\section{Myenteric plexus}

The MP was the only plexus in which the percentages of cholinergic and non-cholinergic $\mathrm{ZnT}^{+}$neurons were almost similar; however, even here the cholinergic $\mathrm{ZnT}^{+}$neurons outnumbered the non-cholinergic $\mathrm{ZnT}^{+}$cells (Table 2). The great majority of the cholinergic $\mathrm{ZnT}^{+}$neurons did not coexpress any of the tested peptides (Table 2). Among the cholinergic $\mathrm{ZnT}^{+}$cells that co-expressed additional peptides, the largest population were those containing NOS (Table 2, Fig. 1d, g). In addition, small percentages of the cholinergic $\mathrm{ZnT}^{+}$neurons co-expressed SOM, VIP, and/or SP (Table 2, Fig. 1e, f). None of the cholinergic $\mathrm{ZnT}^{+}$neurons was ever immunoreactive for GAL (Table 2). Among the noncholinergic $\mathrm{ZnT}^{+}$neurons, almost $50 \%$ were devoid of any of the tested peptides (Table 2). However, a huge subpopulation of the non-cholinergic $\mathrm{ZnT}^{+}$neurons were simultaneously immunoreactive for NOS (Table 2). In addition, small percentages of the non-cholinergic $\mathrm{ZnT}^{+}$neurons coexpressed SOM, VIP, SP, and/or LENK, and single $\mathrm{ZnT3}^{+}$ cells co-expressed NPY (Table 2, Fig. 1h, i). None of the noncholinergic $\mathrm{ZnT}^{+}$neurons was ever found to be immunoreactive for GAL or CGRP (Table 2).

\section{Outer submucosal plexus}

In the OSP, the cholinergic $\mathrm{ZnT}^{+}$cells constituted almost $95 \%$ of the total population of $\mathrm{ZnT}^{+}$neurons (Table 2). Triple-labeling immunofluorescence revealed that a large percentage of the cholinergic $\mathrm{ZnT}^{+}$neurons in the OSP was also immunoreactive for SOM, and many cells coexpressed VIP and/or SP (Table 2, Fig. 2b). In addition, a small percentage of the cholinergic $\mathrm{ZnT}^{+}$neurons coexpressed NOS (Table 2), but no immunoreactivity for GAL was observed (Table 2). The non-cholinergic $\mathrm{ZnT3}^{+}$ neurons in the OSP formed a small subpopulation of $\mathrm{ZnT}^{+}$ cells, and most of them were devoid of any of the tested peptides (Table 2). Only a small percentage of these neurons co-expressed NOS, SOM, VIP, and/or SP, and single $\mathrm{ZnT3}^{+}$ cells co-expressed GAL (Table 2, Fig. 2a). No immunoreactivity for LENK, NPY, or CGRP was observed in these neurons (Table 2, Fig. 2c).

\section{Inner submucosal plexus}

In the ISP, almost all $\mathrm{ZnT3}^{+}$neurons were simultaneously cholinergic (Table 2). Moreover, triple-labeling immunofluorescence revealed that almost half of these cholinergic $\mathrm{ZnT}^{+}$neurons were also GAL-positive, and that many other cells co-expressed SOM, VIP, and SP (Table 2, Fig. $3 \mathrm{a}-\mathrm{c}$ ). On the other hand, none of the cholinergic $\mathrm{ZnT}^{+}$neurons was ever immunoreactive for NOS (Table 2). The subpopulation of non-cholinergic $\mathrm{ZnT}^{+}$neurons in the ISP was even smaller than that in the OSP, and as in the MP and OSP, most of these neurons were devoid of any of the tested peptides (Table 2). Small percentages of the noncholinergic $\mathrm{ZnT}^{+}$neurons co-expressed GAL, SOM, VIP, 

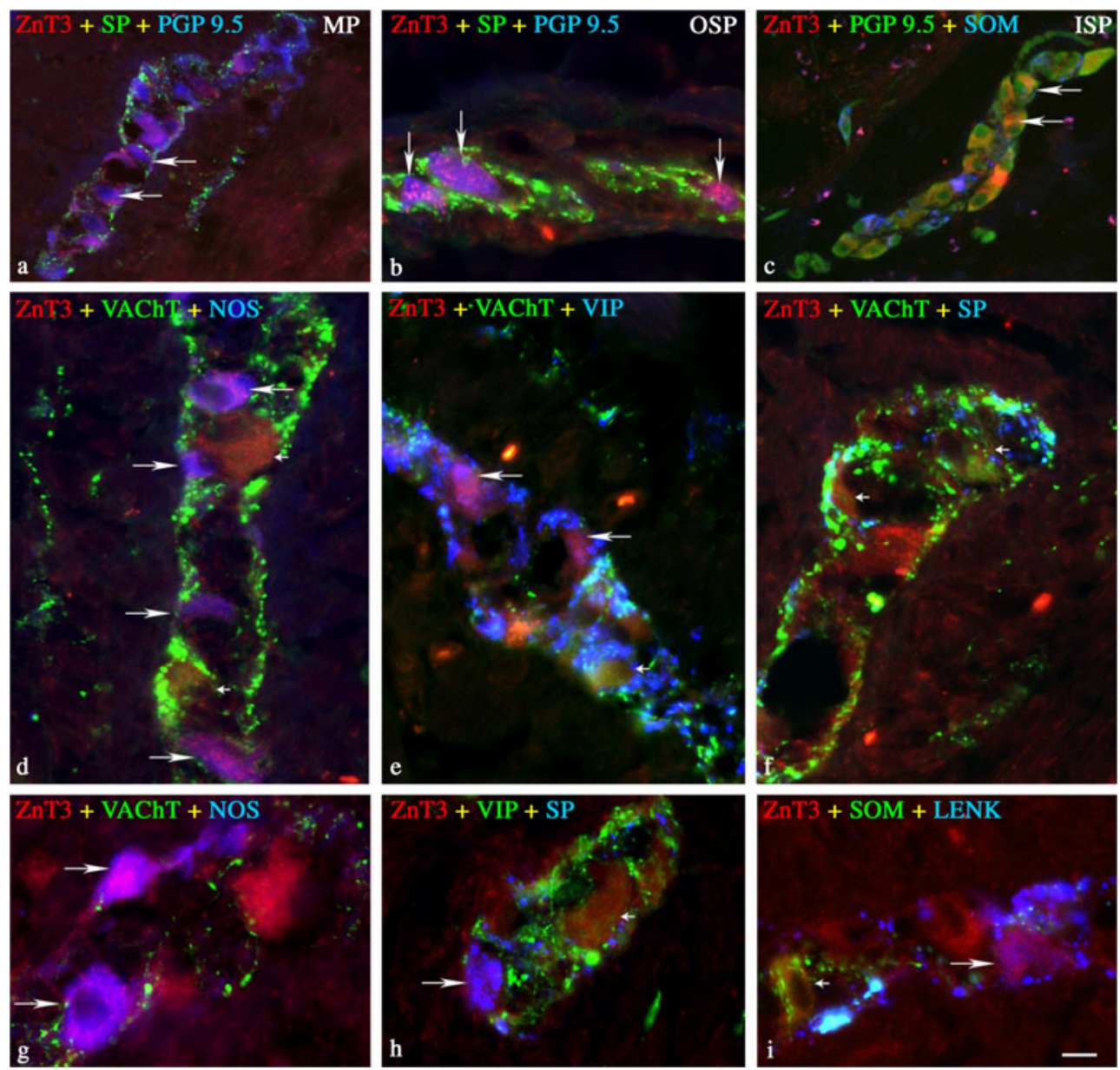

Fig. 1 Representative images of $\mathrm{ZnT}^{+}$neurons located in porcine jejunum. All images are composites of merged images taken separately from blue, red, and green fluorescent channels. a Myenteric plexus $(M P)$; ZnT3 (red) neurons labeled for PGP9.5 (blue) but SP (green)immunonegative (two single arrows). b Outer submucosal plexus $(O S P)$; ZnT3 (red) neurons labeled for PGP9.5 (blue) but SP (green)immunonegative (three single arrows). c Inner submucosal plexus (ISP); ZnT3 (red) neurons labeled for PGP9.5 (green) but SOM (blue)-immunonegative (two single arrows). $\mathbf{d}-\mathbf{i} \mathrm{ZnT}^{+}$neurons located in MP of porcine jejunum. d ZnT3 (red) neuron labeled for NOS (blue) and VAChT (green)-immunonegative (four single arrows), and ZnT3 (red) neurons labeled for VAChT (green) but NOS (blue)-immunonegative (two single small arrows). e ZnT3 (red) neurons labeled for
VIP (blue) but VAChT (green)-immunonegative (two single arrows), and ZnT3 (red) neurons labeled for VAChT (green) but NOS (blue)immunonegative (single small arrow). f ZnT3 (red) neuron labeled for VAChT (green) but SP (blue)-immunonegative (two single small arrows). g ZnT3 (red) neuron labeled for NOS (blue) but VAChT (green)-immunonegative (two single arrows). h ZnT3 (red) neurons labeled for SP (blue) but VIP (green)-immunonegative (single arrow), and ZnT3 (red) neurons labeled for VIP (green) but SP (blue)-immunonegative (single small arrow). i ZnT3 (red) neurons labeled for LENK (blue) but SOM (green)-immunonegative (single arrow), and ZnT3 (red) neurons labeled for SOM (green) but LENK (blue)-immunonegative (single small arrow). Bar $25 \mu \mathrm{m}$
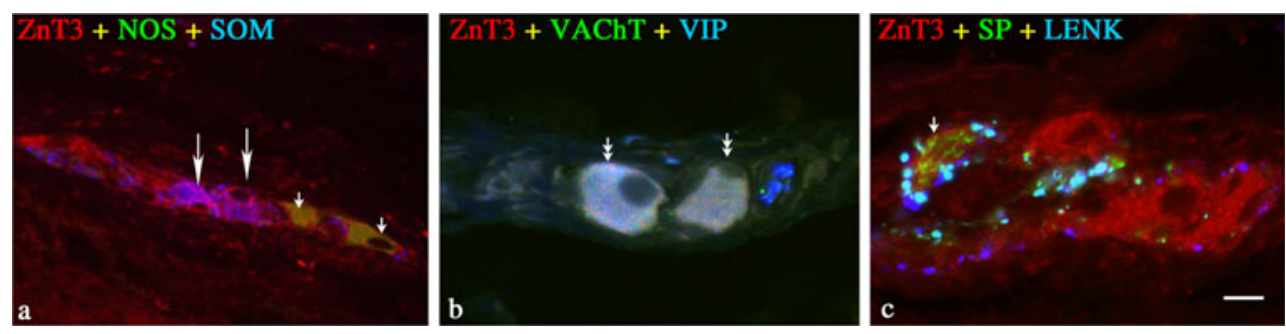

Fig. 2 Representative images of $\mathrm{ZnT}^{+}$neurons located in OSP of porcine jejunum. All images are composites of merged images taken separately from the blue, red, and green fluorescent channels. a ZnT3 (red) neuron labeled for NOS (green) but SOM (blue)-immunonegative (two small single arrows), and $\mathrm{ZnT3}$ (red) neuron labeled for SOM (blue) but NOS (green)-immunonegative (two single arrows). b ZnT3 (red) neuron labeled for VAChT (green) and VIP (blue)-immunopositive (two small double arrows). c ZnT3 (red) neuron labeled for SP (green) and VIP (blue)-immunonegative (small arrow). Bar $25 \mu \mathrm{m}$ 
and/or SP (Table 2, Fig. 3d-f). None of the non-cholinergic $\mathrm{ZnT3}^{+}$neurons co-expressed NOS, LENK, NPY, or CGRP (Table 2).

\section{Discussion}

This is the first report providing a detailed description of the distribution, number, and neurochemical characteristics of the $\mathrm{ZnT}^{+}$neurons in the ENS of porcine jejunum. Numerous cells immunoreactive for $\mathrm{Znt} 3$ have been found within all types of jejunal ganglia, a finding generally consistent with previous investigations on human and porcine large intestine (Gonkowski et al. 2009; Gonkowski 2011). The considerable quantity of $\mathrm{ZnT}^{+}$cells, namely more than $35 \%$ of all enteric neurons in each plexus, and the broadspectrum of active substances that co-localize with this peptide in each plexus suggest that ZnT3 and Zn are important factors within the digestive tract, and that both might be involved in the regulation of various processes in the gut. Moreover, a previous study of porcine large intestine (Gonkowski 2011) has indicated the participation of ZnT3 and $\mathrm{Zn}$ in mechanisms of pathological states within the digestive tract. Although the precise function(s) of ZnT3 neurons and $\mathrm{Zn}$ are currently unknown, their presence in the enteric ganglia (this study; Gonkowski et al. 2009; Gonkowski 2011) and in the adrenergic and cholinergic sympathetic neurons of the murine peripheral nervous system (Wang et al. 2001a, 2003; Wang and Dahlstrom 2008) suggest that they play an integral role in the function of the gut. The majority of work concerning the role of $\mathrm{ZnT3}$ and of $\mathrm{Zn}$ targets has been conducted in the CNS. Previous studies of $\mathrm{Zn}$-containing neurons in the CNS imply that ZnT3 facilitates neuromodulation and/or protects neurons from the cytotoxic action of $\mathrm{Zn}$ during pathological processes (Cousins et al. 2006; Danscher et al. 2003; Kim et al. 2000; Palmiter and Huang 2004; Wang et al. 2003; Wenzel et al. 1997). ZnT3 in particular plays an important part in the regulation of $\mathrm{Zn}$ levels and is the key protein involved with $\mathrm{Zn}$ transport in synaptic vesicles (Danscher et al. 2003; Wenzel et al. 1997). Both ZnT3 and Zn are primarily present in glutamatergic terminals within the CNS (Danscher et al. 2001). However, $\mathrm{Zn}$ is also present in GABA- and glycinecontaining neurons (Danscher et al. 2001; Birinyi et al. 2001; Wang et al. 2001b). In the hippocampus, $\mathrm{Zn}$ is coreleased with glutamate from synapses, where it exerts a strong modulatory effect on N-methyl-D-aspartate (NMDA) receptors (Vogt et al. 2000). Moreover, evidence has been presented that $\mathrm{Zn}$ can modulate both excitatory and inhibitory neurotransmission (Smart et al. 2004). Excitatory NMDA receptors are directly inhibited by Zn, whereas non-NMDA receptors appear relatively unaffected (Paoletti and Neyton 2007; Smart et al. 2004). Because it is released at many glutamatergic synapses, $\mathrm{Zn}$ is likely to be an endogenous allosteric modulator of NMDA receptors (Paoletti and Neyton 2007). Glycinergic (inhibitory) transmission in the CNS might also be affected by $\mathrm{Zn}$, thereby causing potentiation (Betz and Laube 2006; Smart et al. 2004). Low concentrations of $\mathrm{Zn}$ potentiate submaximal glycineinduced currents, whereas higher concentrations cause
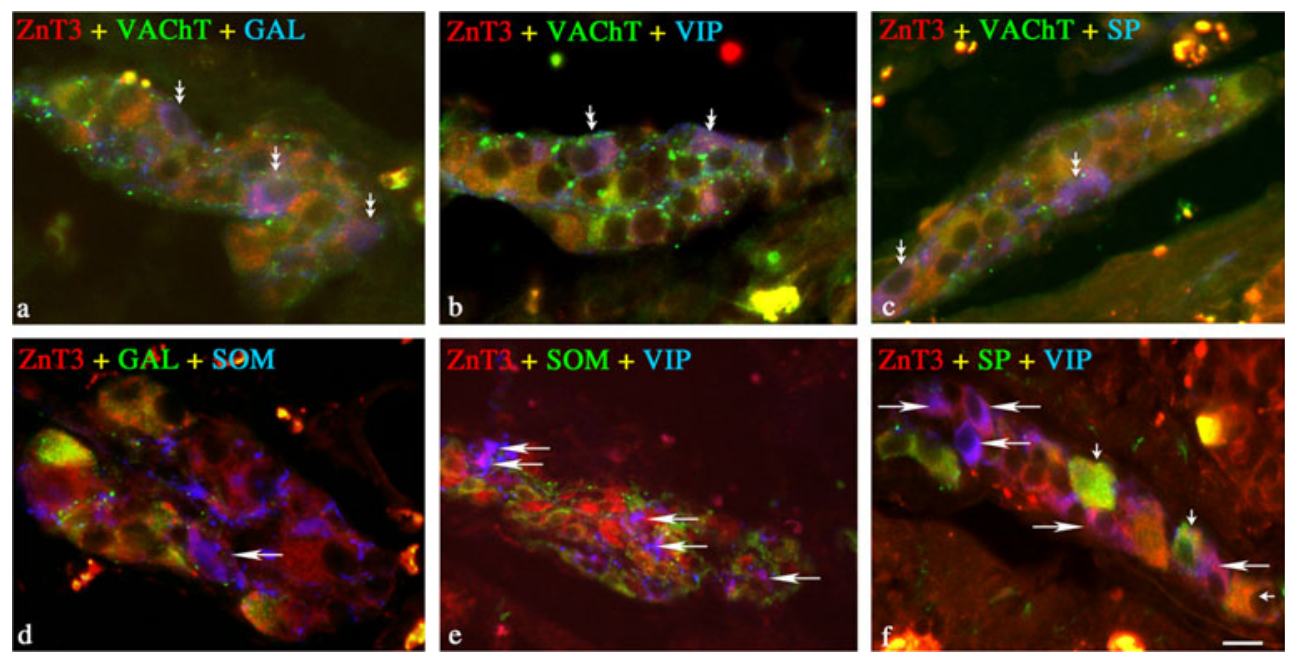

Fig. 3 Representative images of $\mathrm{ZnT}^{+}$neurons located in ISP of porcine jejunum. All images are composites of merged images taken separately from the blue, red, and green fluorescent channels. a ZnT3 (red) neurons labeled for GAL (blue) and VAChT (green)-immunopositive (three double small arrows). b ZnT3 (red) neuron labeled for VIP (blue) and VAChT (green)-immunopositive (two double small arrows). c ZnT3 (red) neurons labeled for VAChT (green) and SP (blue)-immunopositive (two double small arrows). d ZnT3 (red) neuron labeled for SOM (blue) but GAL (green)-immunonegative (single arrow). e ZnT3 (red) neurons labeled for VIP (blue) but SOM (green)-immunonegative (five single arrows). f ZnT3 (red) neuron labeled for VIP (blue) but VAChT (green)-immunonegative (five single arrows), and ZnT3 (red) neurons labeled for VAChT (green) but VIP (blue)-immunonegative (three single small arrows). Bar $25 \mu \mathrm{m}(\mathbf{a}-\mathbf{d}$, f), $100 \mu \mathrm{m}(\mathbf{e})$ 
competitive inhibition (Betz and Laube 2006). A point mutation at the murine Glral locus, which selectively suppresses $\mathrm{Zn}$ potentiation, generates a phenotype that mimics that of patients with hereditary startle disease and thus is indicative of decreased glycinergic inhibition (Betz and Laube 2006). All these data taken together indicate that $\mathrm{Zn}$ is a potent modulator of both excitatory and inhibitory neurotransmission within CNS, and so it might play a similar role in ENS. Such a mechanism might in part explain the co-expression of ZnT3 by so many excitatory cholinergic and inhibitory nitrergic intestinal neurons (present study). In particular, cholinergic receptors in the brain might be directly up- or down-regulated by $\mathrm{Zn}$, whereas other cells types might be modulated via various other types of receptors, such as opioid or catecholamine receptors (Frederickson et al. 2005). Since each of the enteric plexuses has its own unique set of functions (Brown and Timmermans 2004; Furness 2006; Shimizu et al. 2008; Timmermans et al. 2001), the possible roles of $\mathrm{ZnT}^{+}$neurons in each of these plexuses will be discussed separately below.

The MP, which is located between the longitudinal and circular muscle layers, is one of a number of elements that are responsible for the control of digestive motility (Huizinga et al. 2011), and a subpopulation from this plexus also innervates the submucosal plexuses and/or regulates the secretory functions of the gut (Brehmer et al. 1994). Moreover, the long myenteric pathway that activates the submucosal secretomotor neurons projects in parallel with motor and vasodilator reflexes, suggesting that this common pathway coordinates intestinal secretion, blood flow, and motility (Reed and Vanner 2003, 2007). Gut motility is controlled by a subpopulation of cholinergic and nitrergic neurons that mediate the respective contraction and relaxation of the circular and longitudinal muscles (Boeckxstaens et al. 1993; Furness 2006; Lincoln et al. 1997; Porter et al. 1996, 1997; Wood et al. 1999). The majority of $\mathrm{ZnT3}^{+}$ myenteric neurons are also immunoreactive for VAChT and/or NOS. Thus, ZnT3 might be present in both neuron populations that act together in co-ordinated reflexes to facilitate smooth muscle contraction (VAChT neurons) and muscle relaxation (NOS neurons), respectively, in the gut. Although we have also found, among the myenteric $\mathrm{ZnT}^{+}$ neurons, cells immunoreactive for various other well-known markers, such as VIP, SOM, and SP, these subpopulations are extremely small and so will not be discussed in the case of MP.

The neurons of the OSP (located near the internal part of circular muscle layer) and ISP (located on the abluminal side of the muscularis mucosae) innervate the submucosal blood vessels and regulate the secretion and intrinsic sensory pathways of the gut in response to the contents of the lumen (Brehmer et al. 2010). A subpopulation of the OSP neurons might also supply the circular muscle layer of the gut (Scheuermann and Timmermans 1993). The results of the present study suggest that almost half of all neurons located in the OSP and ISP is ZnT3-positive, and almost all of the $\mathrm{ZnT}^{+}$neurons in both these plexuses are simultaneously immunoreactive for VAChT. Such a large population of $\mathrm{ZnT}^{+} / \mathrm{VAChT}^{+}$neurons indicates that $\mathrm{ZnT} 3$ is engaged in various excitatory functions in the OSP and ISP, including muscle contraction in the muscularis mucosa and the increased activity of the mucosal glands (Cooke 2000). Many of the $\mathrm{ZnT}^{+}$neurons in the OSP and ISP have been found to be immunoreactive for SOM, VIP, and/ or SP; this is consistent with previous studies concerning $\mathrm{ZnT}^{+}$neurons in the porcine large intestine (Gonkowski 2011). SOM exerts many effects in the ENS; these include the inhibition of peristalsis (Grider et al. 1987), the regulation of blood flow in the intestines ( $\mathrm{Li}$ et al. 1996), the inhibition of gastrin, cholecystokinin, and VIP (Foong et al. 2010; Low 2004), and the inhibition of extrinsic afferent sensory neuron activity (Furness 2012; Hasler et al. 1993; Plourde et al. 1993). However, the role of ZnT3 in these processes is obscure at present. VIP is strongly co-expressed in $\mathrm{ZnT}^{+}$neurons of both submucosal plexuses, but especially in the ISP. This is congruent with the finding that submucosal VIP neurons are responsible for increasing secretory activity at mucosal glands in the small intestine (Olsson and Holmgren 2001). SP is also an important cotransmitter of cholinergic excitatory motor neurons, and so unsurprisingly, many of the $\mathrm{ZnT}^{+}$neurons immunoreactive for VAChT also co-express SP (Furness 2006; Shimizu et al. 2008). In the OSP and ISP, neurons using SP as their neurotransmitter are thought to be involved mostly in the regulation of the transport and/or secretion of $\mathrm{H}_{2} \mathrm{O}$ and/or electrolytes (Shimizu et al. 2008; Keast et al. 1985), and so $\mathrm{ZnT} 3$ might also be engaged in all these processes. We should add that SP, in both these plexuses, might also be a neurotransmitter and/or neuromodulator of interneurons or cells supplying intestinal blood vessels (Shimizu et al. 2008). The last two substances which are worth mentioning here, are GAL and CGRP. GAL is strongly co-expressed by $\mathrm{ZnT}^{+}$neurons in the ISP, whereas such cells are extremely rare in the OSP (almost half of the $\mathrm{ZnT}^{+}$neurons in the ISP co-express GAL). A similar pattern of GAL co-expression in $\mathrm{ZnT}^{+}$neurons has also been observed in the intramural ganglia of the porcine large intestine (Gonkowski 2011). GAL is often expressed in the ISP by neurons that are engaged in the regulation of the intestinal secretion (Furness 2006) and/or neurotransmitter secretion from other intestinal neurons (Piqueras et al. 2004; Sarnelli et al. 2004). The extensive co-localization of ZnT3and GAL in the ISP neurons suggests that $\mathrm{ZnT3}$ and $\mathrm{Zn}$ are involved in some of these processes. CGRP is a marker peptide for Dogiel type II neurons, which are putative intrinsic primary afferent neurons (Timmermans et al. 1997; Wolf et al. 2007). 
However, some recent studies have shown CGRP-positive neurons displaying a distinctly different morphology (Wolf et al. 2007). Although CGRP-containing neurons have been observed in the present study, and although most of them have the features of Dogiel type II neurons, these cells are ZnT3-negative. Thus, detectable ZnT3 immunoreactivity might not occur in intestinal intrinsic sensory neurons.

In conclusion, $\mathrm{ZnT}^{+}$neurons have been identified in enteric ganglia of the porcine jejunum. A broad-spectrum of neuroactive substances has been found to co-localize with $\mathrm{ZnT3}$, suggesting that $\mathrm{ZnT3}$ neurons are involved in many of the functional processes throughout the length of the jejunum. Further studies are required to elucidate the role of these neurons during pathological states of the jejunum.

Acknowledgments The authors are grateful for the primary antibody (against zinc transporter 3) provided as a kind gift by Prof. Richard Palmiter (Howard Hughes Medical Institute and Department of Biochemistry, University of Washington, USA).

Open Access This article is distributed under the terms of the Creative Commons Attribution License which permits any use, distribution, and reproduction in any medium, provided the original author(s) and the source are credited.

\section{References}

Betz H, Laube B (2006) Glycine receptors: recent insights into their structural organization and functional diversity. J Neurochem 97:1600-1610

Birinyi A, Parker D, Antal M, Shupliakov O (2001) Zinc co-localizes with GABA and glycine in synapses in the lamprey spinal cord. J Comp Neurol 433:208-221

Boeckxstaens GE, Pelckmans PA, Herman AG, Van Maercke YM (1993) Involvement of nitric oxide in the inhibitory innervation of the human isolated colon. Gastroenterology 104:690-697

Brehmer A, Stach W, Addicks K (1994) Fine structural distinction between ganglia of the outer and inner submucosal plexus in porcine small intestine. Acta Anat (Basel) 151:188-193

Brehmer A, Rupprecht H, Neuhuber W (2010) Two submucosal nerve plexus in human intestines. Histochem Cell Biol 133:149-161

Brown DR, Timmermans JP (2004) Lessons from the porcine enteric nervous system. Neurogastroenterol Motil 16:50-54

Cooke HJ (2000) Neurotransmitters in neuronal reflexes regulating intestinal secretion. Ann N Y Acad Sci 915:77-80

Cousins RJ, Liuzzi JP, Lichten LA (2006) Mammalian zinc transport, trafficking, and signals. J Biol Chem 281:24085-24089

Danscher G, Jo SM, Varea E, Wang Z, Cole TB, Schroder HD (2001) Inhibitory zinc-enriched terminals in mouse spinal cord. Neuroscience 105:941-947

Danscher G, Wang Z, Kim YK, Kim SJ, Sun Y, Jo SM (2003) Immunocytochemical localization of zinc transporter 3 in the ependyma of the mouse spinal cord. Neurosci Lett 342:81-84

Foong JP, Parry LJ, Gwynne RM, Bornstein JC (2010) 5-HT(1A), SST (1), and SST(2) receptors mediate inhibitory postsynaptic potentials in the submucous plexus of the guinea pig ileum. Am J Physiol Gastrointest Liver Physiol 298:G384-G394

Frederickson CJ, Suh SW, Silva D, Frederickson CJ, Thompson RB (2000) Importance of zinc in the central nervous system: the zinccontaining neuron. J Nutr 130:1471S-1483S
Frederickson CJ, Koh JY, Bush AI (2005) The neurobiology of zinc in health and disease. Nat Rev Neurosci 6:449-462

Furness JB (2000) Types of neurons in the enteric nervous system. J Auton Nerv Syst 81:87-96

Furness JB (2006) The enteric nervous system. Blackwell, Oxford

Furness JB (2012) The enteric nervous system and neurogastroenterology. Nat Rev Gastroenterol Hepatol 9:286-294

Gonkowski S (2011) Neurochemical characterization of zinc transporter 3-immunoreactive (ZnT3-IR) neurons of enteric neuronal system in porcine large intestine and its plasticity during inflamation and after axotomy within descending colon. University Warmia and Mazury in Olsztyn, ISBN 978-83-7299-744-9 (in Polish). Thesis for degree of associate professor

Gonkowski S, Kaminska B, Landowski P, Skobowiat C, Burlinski P, Majewski M, Calka J (2009) A population of zinc transporter 3like immunoreactive neurons is present in the ganglia of human descending colon. Adv Clin Exp Exp Med 18:243-248

Grider JR, Arimura A, Makhlouf GM (1987) Role of somatostatin neurons in intestinal peristalsis: facilitatory interneurons in descending pathways. Am J Physiol 253:G434-G438

Hasler WL, Soudah HC, Owyang C (1993) A somatostatin analogue inhibits afferent pathways mediating perception of rectal distention. Gastroenterology 104:1390-1397

Huizinga JD, Martz S, Gil V, Wang XY, Jimenez M, Parsons S (2011) Two independent networks of interstitial cells of Cajal work cooperatively with the enteric nervous system to create colonic motor patterns. Front Neurosci 5:93

Jo SM, Danscher G, Daa SH, Won MH, Cole TB (2000) Zinc-enriched (ZEN) terminals in mouse spinal cord: immunohistochemistry and autometallography. Brain Res 870:163-169

Keast JR, Furness JB, Costa M (1985) Different substance P receptors are found on mucosal epithelial cells and submucous neurons of the guinea-pig small intestine. Naunyn Schmiedebergs Arch Pharmacol 329:382-387

Kim AH, Sheline CT, Tian M, Higashi T, McMahon RJ, Cousins RJ, Choi DW (2000) L-type $\mathrm{Ca}(2+)$ channel-mediated $\mathrm{Zn}(2+)$ toxicity and modulation by ZnT-1 in PC12 cells. Brain Res 886 : 99-107

Li MK, Sung JJ, Woo KS, Sanderson J, Leung NW, Yu LM, Tsui CP, Chung SC, Leung FW (1996) Somatostatin reduces gastric mucosal blood flow in patients with portal hypertensive gastropathy: a randomized, double-blind crossover study. Dig Dis Sci 41: 2440-2446

Lincoln J, Hoyle CH, Cafri G (1997) Nitric oxide in health and disease. Cambridge University Press, Cambridge

Low MJ (2004) Clinical endocrinology and metabolism. The somatostatin neuroendocrine system: physiology and clinical relevance in gastrointestinal and pancreatic disorders. Best Pract Res Clin Endocrinol Metab 18:607-622

Olsson C, Holmgren S (2001) The control of gut motility. Comp Biochem Physiol A Mol Integr Physiol 128:481-503

Palmiter RD, Huang L (2004) Efflux and compartmentalization of zinc by members of the SLC30 family of solute carriers. Pflugers Arch 447:744-751

Palmiter RD, Cole TB, Quaife CJ, Findley SD (1996) ZnT-3, a putative transporter of zinc into synaptic vesicles. Proc Natl Acad Sci USA 93:14934-14939

Paoletti P, Neyton J (2007) NMDA receptor subunits: function and pharmacology. Curr Opin Pharmacol 7:39-47

Piqueras L, Tache Y, Martinez V (2004) Galanin inhibits gastric acid secretion through a somatostatin-independent mechanism in mice. Peptides 25:1287-1295

Plourde V, Lembo T, Shui Z, Parker J, Mertz H, Tache Y, Sytnik B, Mayer E (1993) Effects of the somatostatin analogue octreotide on rectal afferent nerves in humans. Am J Physiol 265:G742G751 
Porter AJ, Wattchow DA, Brookes SJ, Schemann M, Costa M (1996) Choline acetyltransferase immunoreactivity in the human small and large intestine. Gastroenterology 111:401-408

Porter AJ, Wattchow DA, Brookes SJ, Costa M (1997) The neurochemical coding and projections of circular muscle motor neurons in the human colon. Gastroenterology 113:1916-1923

Reed DE, Vanner SJ (2003) Long vasodilator reflexes projecting through the myenteric plexus in guinea-pig ileum. J Physiol (Lond) 553:911-924

Reed DE, Vanner S (2007) Mucosal stimulation activates secretomotor neurons via long myenteric pathways in guinea pig ileum. Am J Physiol Gastrointest Liver Physiol 292:G608-G614

Sarnelli G, Vanden BP, Raeymaekers P, Janssens J, Tack J (2004) Inhibitory effects of galanin on evoked $\left[\mathrm{Ca}^{2+}\right]_{\mathrm{i}}$ responses in cultured myenteric neurons. Am J Physiol Gastrointest Liver Physiol 286:G1009-G1014

Scheuermann DW, Timmermans JP (1993) Differing chemical content of the neuronal populations of submucosal ganglionic plexus of the enteric nervous system. Gastroenterology 104:1579

Shimizu Y, Matsuyama H, Shiina T, Takewaki T, Furness JB (2008) Tachykinins and their functions in the gastrointestinal tract. Cell Mol Life Sci 65:295-311

Smart TG, Hosie AM, Miller PS (2004) $\mathrm{Zn}^{2+}$ ions: modulators of excitatory and inhibitory synaptic activity. Neuroscientist 10:432-442

Timmermans JP, Adriaensen D, Cornelissen W, Scheuermann DW (1997) Structural organization and neuropeptide distribution in the mammalian enteric nervous system, with special attention to those components involved in mucosal reflexes. Comp Biochem Physiol A Physiol 118:331-340
Timmermans JP, Hens J, Adriaensen D (2001) Outer submucous plexus: an intrinsic nerve network involved in both secretory and motility processes in the intestine of large mammals and humans. Anat Rec 262:71-78

Vogt K, Mellor J, Tong G, Nicoll R (2000) The actions of synaptically released zinc at hippocampal mossy fiber synapses. Neuron 26:187-196

Wang ZY, Dahlstrom A (2008) Axonal transport of zinc transporter 3 and zinc containing organelles in the rodent adrenergic system. Neurochem Res 33:2472-2479

Wang Z, Danscher G, Mook JS, Shi Y, Daa SH (2001a) Retrograde tracing of zinc-enriched (ZEN) neuronal somata in rat spinal cord. Brain Res 900:80-87

Wang Z, Li JY, Dahlstrom A, Danscher G (2001b) Zinc-enriched GABAergic terminals in mouse spinal cord. Brain Res 921:165-172

Wang ZY, Danscher G, Dahlstrom A, Li JY (2003) Zinc transporter 3 and zinc ions in the rodent superior cervical ganglion neurons. Neuroscience 120:605-616

Wenzel HJ, Cole TB, Born DE, Schwartzkroin PA, Palmiter RD (1997) Ultrastructural localization of zinc transporter-3 (ZnT-3) to synaptic vesicle membranes within mossy fiber boutons in the hippocampus of mouse and monkey. Proc Natl Acad Sci USA 94:12676-12681

Wolf M, Schrodl F, Neuhuber W, Brehmer A (2007) Calcitonin generelated peptide: a marker for putative primary afferent neurons in the pig small intestinal myenteric plexus? Anat Rec (Hoboken) 290:1273-1279

Wood JD, Alpers DH, Andrews PL (1999) Fundamentals of neurogastroenterology. Gut 45 (Suppl 2):II6-II16 